\title{
Energy-Efficient Industrial Internet of UAVs for Power Line Inspection in Smart Grid
}

\author{
Zhenyu Zhou, Senior Member, IEEE, Chuntian Zhang, Chen Xu, Member, IEEE, Fei Xiong, Yan Zhang, Senior \\ Member, IEEE, Tariq Umer, Senior Member, IEEE,
}

\begin{abstract}
Industrial internet of unmanned aerial vehicles (IIoUAVs) which enable autonomous inspection and measurement of anything anytime anywhere have become an essential component of the future industrial internet of things (IIoT) ecosystem. In this paper, we investigate how to apply IIoUAVs for power line inspection in smart grid from an energy efficiency perspective. Firstly, the energy consumption minimization problem is formulated as a joint optimization problem, which involves both the large-timescale optimization such as trajectory scheduling, velocity control, and frequency regulation, and the small-timescale optimization such as relay selection and power allocation. Then, the original NP-hard problem is transformed into a two-stage suboptimal problem by exploring the timescale difference and the energy magnitude difference between the large-timescale and the small-timescale optimizations, and is solved by combining dynamic programming (DP), auction theory and matching theory. Finally, the proposed algorithm is verified based on real-world map and realistic power grid topology.
\end{abstract}

Index Terms-IIoUAVs, IIoT, power line inspection, energy efficiency, dynamic programming, auction theory, matching theory, smart grid.

\section{INTRODUCTION}

\section{A. Background and Motivation}

W ITH the evolutionary development of the smart grid industry, it is estimated that by 2020 , the total length of transmission power lines in US will increase to 0.59 million kilometers with a compound annual growth rate of $4 \%$, while the global annual growth rate is $3 \%$ [1]. This massive deployment poses new challenges on maintenance and inspection since power lines deployed in the fields are subject to numerous adverse environmental and human impacts such as wind blowing, sun exposure, rain erosion, etc [2], [3]. Accordingly, power line inspection which allows fast detection of a series of defects including line damage, cracking,

Manuscript received September 30, 2017; revised November 07, 2017; accepted December 28, 2017.

This work was partially supported by the National Science Foundation of China (NSFC) under Grant Numbers 61601181, the Fundamental Research Funds for the Central Universities under Grant Numbers 2017MS13; supported by Beijing Natural Science Foundation (4174104); Beijing Outstanding Young Talent under Grant Number 2016000020124G081.

Z. Zhou, C. Zhang, C. Xu and F. Xiong are with the State Key Laboratory of Alternate Electrical Power System with Renewable Energy Sources, School of Electrical and Electronic Engineering, North China Electric Power University, Beijing, China, 102206. C. Xu is the corresponding author. (e-mail: zhenyu_zhou@ncepu.edu.cn, chuntianzhang_1994@163.com, chen.xu@ncepu.edu.cn, fei.xiong@ncepu.edu.cn).

Y. Zhang is with the Department of Informatics, University of Oslo, Norway, and also with the Simula Research Laboratory, Norway (e-mail: yanzhang@ieee.org).

T. Umer is with the Department of Computer Science COMSATS Institute of Information Technology, Wah Cantt. (e-mail: t.umer@ciitwah.edu.pk). galvanization loss, corrosion, and insulating breakage, is of significant importance for preventative maintenance [4]. At present, power line inspection is mainly conducted via human patrol, which exhibits the following major problems [5]: 1) Safety issues: the electromagnetic field radiation generated by high-voltage power lines with hundreds of kilovolts is hazardous to human health; 2) Accuracy issues: high inspection accuracy cannot be guaranteed due to large-scale distribution and complex geographical environment; 3) Cost issues: the labor, transportation, and equipment costs can be enormously high. Thus, a shift of paradigm is urgently required for the development of automatic machine-based inspection approaches with excellent cost-performance balance.

To this end, industrial unmanned aerial vehicles (UAVs), which enable autonomous inspection and measurement of anything anytime anywhere, have emerged as a promising solution for power line inspection. Due to the unique characteristics such as fast deployment, easy programmability, reconfiguration, control flexibility, and scalability [6], industrial internet of UAVs (IIoUAVs) which enable ubiquitous information sharing and fine-granularity coordination among a fleet of UAVs with little or no human intervention, are essential to realize the framework of future industrial internet of things (IIoT) ecosystem [7]. During inspection, IIoUAVs mounted with up-to-date visual inspection and communication devices can consecutively capture high-resolution images of power lines and deliver the data back to ground base stations (BSs) [8].

Despite the huge potential benefits of IloUAVs, the endurance and reliability performance is fundamentally limited by the maximum battery capacity, which is generally small due to practical space, cost and weight constraints [9]. For an instance, the battery capacity of a small-type UAV with a payload of 300 grams is only around 5200mAh, which is equivalent to eight alkaline batteries [10]. The energy consumption model of IIoUAVs is also different from conventional terrestrial communication systems. On one hand, since power line inspection is delay tolerant, inspection data are firstly stored in local storage devices temporarily, and are then delivered to the remote BS via some relay UAVs in an opportunistic multi-hop fashion [11]. The optimization of relay selection and power allocation depends on channel variations in the timescale of milliseconds. On the other hand, additional energy consumption is required for IIoUAVs to support mobility and avoid collision, which is usually several orders of magnitudes higher than the energy consumed for data delivery [12], and relies on trajectory variations in the 
timescale of seconds.

Therefore, how to achieve energy-efficient IIoUAVs for power line inspection remains nontrivial. Firstly, it is difficult to determine the optimal energy minimization strategy subject to the complicated power grid topology, UAV flying model, and various practical environmental factors. Secondly, it is infeasible to derive a polynomial-time solution since the largetimescale optimization variables are coupled with the smalltimescale optimization variables. Last but not least, the energy consumption performance should be evaluated in a trustworthy manner based on realistic power grid topology data.

\section{B. Contribution}

We summarize the main contributions of this paper as follows.

- We investigate how to realize energy-efficient IIoUAVs for power line inspection in smart grid. The energy consumption minimization problem is formulated as a joint optimization problem, which involves both the largetimescale optimization such as trajectory scheduling, velocity control, and frequency regulation, and the smalltimescale optimization such as relay selection and power allocation. The formulated problem is NP-hard due to the coupling between the large-timescale and small-timescale optimization variables.

- We explore both the timescale difference and the energy magnitude difference to transform the NP-hard problem into a two-stage suboptimal optimization problem, in which the large-timescale optimization problem is solved in the first stage by dynamic programming (DP), and the small-timescale optimization problem is solved in the second stage by combining auction theory and matching theory.

- We evaluate the energy consumption performance based on real-world map and realistic power grid topology. The relationships between energy consumption and other key parameters, including the total number of target points, broadcasting frequency, velocity and computational complexity are illustrated through numerical results.

The structure of this work is elaborated as follows. A comprehensive review of the state-of-the-art development is provided in Section II. The system model with the explicit consideration of the energy consumed for propulsion, collision avoidance, and image data delivery is presented in Section III. The formulated joint optimization problem is developed in Section IV. The two-stage energy-efficient power line inspection algorithm is described in Section V. Numerical results and related analysis are presented in Section VI. Section VII concludes this paper and identifies future directions.

\section{State Of The ART}

Note that the power line inspection by utilizing UAVs has been already studied recently [6], [13]-[16]. In [6], the genetic algorithm (GA) was proposed to obtain effective line inspection path which supports the shortest length. An altitude control system for UAV visual inspection was presented in [13], which was designed based on quaternions algebra. The authors in [14] studied a method for transmission line inspection and tracking by employing Hough transform and Kalman filter. In [15], a power line inspection algorithm called PLineD was proposed to address the real-time perception problem. A trajectory scheduling approach for UAVs in a complex 3D environment was designed in [16] by combining GA and particle swarm optimization to minimize the execution time. These works mainly focus on how to utilize UAVs to realize power line detection system, but have not taken into account the energy consumption of UAVs and the communication resource allocation problems.

Energy-efficiency problems of UAVs have been studied in [11], [12], [17]-[20]. In [11], the network energy efficiency was maximized through the joint optimization of the speed and load of UAVs which act as communication relays for ground nodes. In [12], the deployment of multi-UAVs was optimized to minimize the flight time while considering the energy constraint. In [17], the energy-efficient communication between UAVs and a ground terminal was investigated via UAV trajectory optimization with the joint consideration of communication throughput and UAV energy consumption. The authors in [18] proposed an energy-efficient resource allocation scheme for UAVs which work as aerial BSs to serve ground devices. A reference small-UAV model called "averaged model" was proposed in [19], and the aircraft stability and the power consumption issues were addressed simultaneously. An energy optimization approach for solarpowered UAVs was proposed in [20], in which the trajectory was optimized by controlling the flight attitude.

It is noted that all above mentioned works mainly study energy-efficiency problems of UAVs in regular wireless communication services that involve air-to-ground links, and cannot be applied for IIoUAVs-based power line inspection. The system models and problem formulations are completely different from our work, since there still lacks a comprehensive consideration of joint trajectory scheduling, frequency regulation, velocity control, communication relay selection and power allocation optimization from an energy efficiency perspective. In this paper, we use DP with a velocity control and frequency regulation sub-process in combination with matching theory and auction theory to optimize energy consumption of propulsion, collision avoidance, and image data delivery for IIoUAVs.

\section{SySTEM MODEL}

The IloUAVs-based power line inspection model is presented in Fig. 1. The power grid topology can be considered as a graph. Let $\mathcal{K}=\{0, \cdots, k, \cdots, K\}$ be the set of nodes of the graph, the elements of which represent the inspection target points such as power towers, renewable energy generators, etc. Let $l_{k, k^{\prime}}$ represent the power line segment between target point $k$ and target point $k^{\prime}$, i.e., $k, k^{\prime} \in \mathcal{K}, k \neq k^{\prime}$. The length of segment $l_{k, k^{\prime}}$ is denoted as $d_{k, k^{\prime}}$, i.e., $d_{k, k^{\prime}}=\infty$ if $k$ and $k^{\prime}$ are disconnected. A fleet of $N$ inspection UAVs (denoted as IUAVs) start from target point $k=0$, then fly along the power lines to carry out both target point inspection and line corridor inspection missions, and finally return back to the 


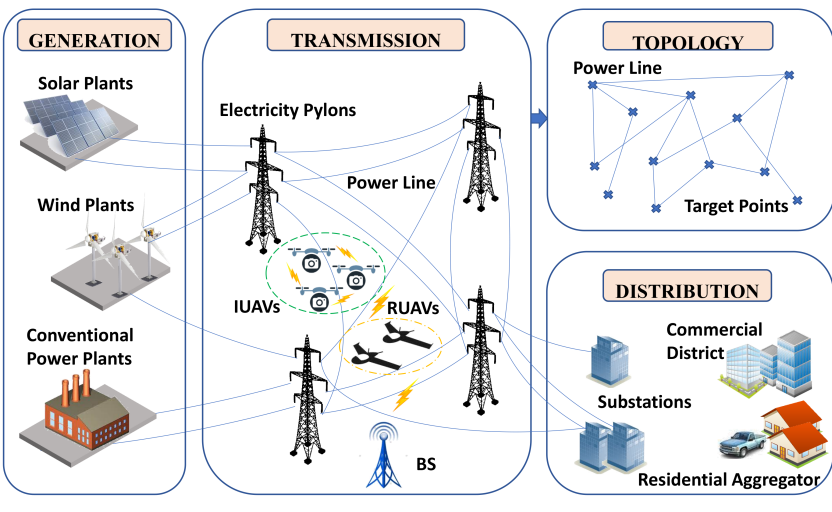

Fig. 1: The IIoUAVs-based power line inspection model.

starting point. It is noted that the flying trajectory of IUAVs must pass through all of the target points.

During inspection, IUAVs form a cluster around the power line and fly forwards with the same velocity in order to provide a multi-angle inspection of the same location within the same timestamp. The communication devices mounted on each IUAV have twofold functions: collision avoidance and image data delivery. On one hand, a number of predefined beacons are broadcasted per second by each IUAV for received signal strength (RSS) based distance measurement. Then, numerous well-knwon collision avoidance schemes [21], [22] can be used to maintain a minimum safety distance between any two IUAVs. On the other hand, a store-carry-forward-based data transmission approach is adopted, in which the data collected by the IUAVs are firstly stored in local storage devices temporarily, and are then delivered to the remote base stations via some relay UAVs (denoted as RUAVs) in an opportunistic multi-hop fashion. Afterwards, the data will be transmitted to the server of grid company, which are then processed and analyzed to identify potential problems. Therefore, the data transmission is delay tolerant. As for optimizing the data delivery latency, we will consider it in our future work. It is noted that RUAVs which are carrying out other tasks and happen to appear within the proximity of IUAVs are not always available. The sets of IUAVs and RUAVs are denoted as $\mathcal{N}=\{1, \cdots, n, \cdots, N\}$, and $\mathcal{M}=\{1, \cdots, m, \cdots, M\}$, respectively.

Hence, the total energy consumption of each IUAV includes three parts, i.e., the amount of energy consumed for propulsion, collision avoidance beacon broadcasting, and image data delivery, the models of which are elaborated in the following subsections.

\section{A. Propulsion Energy Consumption Model}

The steady straight-and-level flight (SSLF) model proposed in [17] is adopted to develop the propulsion energy consumption model. The SSLF model implies the following two aspects: 1) an IUAV flies towards a fixed direction with a constant speed without horizontal acceleration and sudden turning; 2) due to the lift-weight balance, an IUAV flies at a constant altitude without vertical acceleration. Defining the velocity of IUAV at segment $l_{k, k^{\prime}}$ as $v_{k, k^{\prime}}$, the propulsion energy consumption required for flying through $l_{k, k^{\prime}}$ is given by

$$
E_{k, k^{\prime}}^{p}\left(v_{k, k^{\prime}}\right)=\frac{d_{k, k^{\prime}}}{v_{k, k^{\prime}}} P_{k, k^{\prime}}^{p}\left(v_{k, k^{\prime}}\right)=d_{k, k^{\prime}} \frac{c_{k, k^{\prime}}^{1}\left(v_{k, k^{\prime}}\right)^{4}+c_{k, k^{\prime}}^{2}}{\left(v_{k, k^{\prime}}\right)^{2}} .
$$

Here $P_{k, k^{\prime}}^{p}\left(v_{k, k^{\prime}}\right)$ is the propulsion power, which is given by

$$
P_{k, k^{\prime}}^{p}\left(v_{k, k^{\prime}}\right)=c_{k, k^{\prime}}^{1}\left(v_{k, k^{\prime}}\right)^{3}+\frac{c_{k, k^{\prime}}^{2}}{v_{k, k^{\prime}}},
$$

where the first term $c_{k, k^{\prime}}^{1}\left(v_{k, k^{\prime}}\right)^{3}$ represents the parasitic power required for balancing the parasitic drag caused by IUAV skin friction, the second term $\frac{c_{k, k^{\prime}}^{2}}{v_{k, k^{\prime}}}$ represents the induced power required for balancing the drag force caused by air redirection. $c_{k, k^{\prime}}^{1}$ and $c_{k, k^{\prime}}^{2}$ are given by

$$
\begin{aligned}
& c_{k, k^{\prime}}^{1} \triangleq \frac{1}{2} \rho_{k, k^{\prime}} C_{k, k^{\prime}}^{d} S, \\
& c_{k, k^{\prime}}^{2} \triangleq \frac{2 W^{2}}{\left(\pi e \eta_{k, k^{\prime}}\right) \rho_{k, k^{\prime}} S},
\end{aligned}
$$

where $\rho_{k, k^{\prime}}$ is the air density, $C_{k, k^{\prime}}^{d}$ is the coefficient of zerolift drag force, $S$ is the reference area of IUAV, $e$ is the Oswald efficiency, and $\eta_{k, k^{\prime}}$ is the wing aspect ratio, and $W$ is the weight of IUAV.

Furthermore, we extend the SSLF model to the more generalized scenario to include the energy consumed by IUAVs at target points for head direction adjustment. Hence, the energy required for adjusting head direction at target point $k^{\prime}$ can be expressed as $\frac{c_{k, k^{\prime}}^{2}}{v_{k, k^{\prime}} g^{2}} \int_{0}^{T} a^{2}(t) d t$, where $a(t)$ is the acceleration of IUAV when adjusting the head direction, $T$ is the duration required for adjusting the head direction, and $g$ is the gravitational acceleration with nominal value $9.8 \mathrm{~m} / \mathrm{s}^{2}$. It is easy to obtain the energy required for head direction adjustment at target point $k^{\prime}$ accounts for only $0.12 \%$ of the energy consumed for flying through segment $l_{k, k^{\prime}}$, which can be neglected for the purpose of simplicity.

\section{B. Collision Avoidance Energy Consumption Model}

The collision avoidance scheme is composed of three stages, i.e., distance estimation, collision warning, and control actions. Here, we only consider the energy consumption of distance estimation, since collision avoidance control rules and actions are out of the scope of this work, and have been intensively studied in [21], [22]. During the flight in segment $l_{k, k^{\prime}}$, an IUAV has to continuously broadcast beacons for inter-IUAV distance estimation with a frequency of $f_{k, k^{\prime}}$, i.e., there are a total of $f_{k, k^{\prime}}$ independent measurements available per second. Considering the imperfect estimation caused by noise, define $\delta_{k, k^{\prime}}$ as the maximum estimation error of distance that can be compensated by collision warning and control actions for successful collision avoidance. The probability that an estimation error is greater than $\delta_{k, k^{\prime}}$ is defined as $\operatorname{Pr}\left(\delta_{k, k^{\prime}}\right)$, which is given by

$$
\operatorname{Pr}\left(\delta_{k, k^{\prime}}\right)=P\left(\text { single estimation error }>\delta_{k, k^{\prime}}\right) .
$$


Hence, a collision occurs if all of the $f_{k, k^{\prime}}$ independent estimations have errors greater than $\delta_{k, k^{\prime}}$, the probability of which is given by

$$
\operatorname{Pr}^{c}\left(f_{k, k^{\prime}}\right)=\operatorname{Pr}\left(\delta_{k, k^{\prime}}\right)^{f_{k, k^{\prime}}} .
$$

It is noted that $\operatorname{Pr}^{c}\left(f_{k, k^{\prime}}\right)$ is also the collision probability. For an instance, assuming that $90 \%$ measurements have an estimation error less than 4 meters, we have $P_{r}(4)=10 \%$. Let $f_{k, k^{\prime}}=4$, we can obtain $\operatorname{Pr}^{c}(4)=10^{-4}$, which represents that the collision probability of any two IUAVs is $10^{-4}$.

When the estimated distance is less than the minimum safety distance, a warning signal is generated and sent to the control unit, which then issues a collision avoidance control action, e.g., heading direction adjustment. The energy consumed for broadcasting beacons is calculated as

$$
E_{k, k^{\prime}}^{c}\left(f_{k, k^{\prime}}, v_{k, k^{\prime}}\right)=\frac{P^{c} d_{k, k^{\prime}} f_{k, k^{\prime}} t_{c}}{v_{k, k^{\prime}}},
$$

where $P^{c}$ and $t_{c}$ denote the power and duration of each beacon signal.

\section{Data Delivery Energy Consumption Model}

We assume that $M_{k, k^{\prime}}$ RUAVs are available when $N$ IUAVs are flying through power line segment $l_{k, k^{\prime}}$, and RUAV $m$ serves as the relay station for IUAV $n$. Data are actually delivered from IUAV $n$ to the BS $B_{0}$ via a two-hop fashion, i.e., data are firstly sent from IUAV $n$ to RUAV $m$ in the first hop, and then are forwarded from RUAV $m$ to BS $B_{0}$ in the second hop. In this work, the one-to-one correspondence mapping between IUAVs and RUAVs is assumed for the purpose of simplicity. The more complex many-to-one matching model will be considered in our future works. Furthermore, we have adopted a simplified model where each UAV occupies an orthogonal frequency band, and thus, the co-channel interference caused by spectrum sharing can be neglected. Similar data transmission models based on orthogonal resource blocks have also been employed in previous works such as [23], [24]. The effective SNR of the two-hop link, i.e., (IUAV $n$, RUAV $m$, $\mathrm{BS} B_{0}$ ), is expressed as

$$
\gamma_{n, m, B_{0}}=\frac{\gamma_{n, m} \gamma_{m, B_{0}}}{\gamma_{n, m}+\gamma_{m, B_{0}}+1} .
$$

Here, $\gamma_{n, m}$ and $\gamma_{m, B_{0}}$ denote the SNR expressions of the firsthop and second-hop links, respectively, which are calculated as

$$
\begin{aligned}
\gamma_{n, m} & =\frac{P_{n}^{d}[t] g_{n, m}}{N_{0}}, \\
\gamma_{m, B_{0}} & =\frac{P_{m}^{d}[t] g_{m, B_{0}}}{N_{0}},
\end{aligned}
$$

where $P_{n}^{d}$ and $P_{m}^{d}$ denote the transmission power of IUAV $n$ and RUAV $m$ for data delivery, respectively. $g_{n, m}$ and $g_{m, B_{0}}$ represent the channel gain between IUAV $n$ and RUAV $m$, and the channel gain between RUAV $m$ and BS $B_{0}$, respectively. $N_{0}$ denotes the one-sided power spectral density of the additive white Gaussian noise (AWGN).
Remark 1: How to derive (7) is omitted here due to space limitations. A similar proof can be found in [25], [26] and references therein.

The energy consumption of data delivery for IUAV $n$ at time slot $t$ is calculated as

$$
E_{k, k^{\prime}}^{n, d}\left(P_{n}^{d}[t]\right)=P_{n}^{d}[t] T_{s},
$$

where $T_{s}$ is the channel coherence time which is negatively proportional to velocity, i.e., $T_{s} \approx c_{3} / v_{k, k^{\prime}}$, Q1-6: where $c_{3}$ is a constant. The total number of time slots at segment $l_{k, k^{\prime}}$ can be calculated as $N_{k, k^{\prime}}^{s}=\left\lfloor d_{k, k^{\prime}} /\left(v_{k, k^{\prime}} T_{s}\right)\right\rfloor$. The set of time slots is defined as $\mathcal{T}_{s}=\left\{1, \cdots, t, \cdots, N_{k, k^{\prime}}^{s} \mid k, k^{\prime} \in \mathcal{K}, k \neq\right.$ $\left.k^{\prime}\right\}$.

\section{Problem Formulation}

Given the power grid topology, the key research challenge is how to jointly determine flying trajectory, velocity, beacon transmission frequency, relay selection, and data transmission power for each IUAV from an energy efficiency perspective under various practical constraints. We use a binary value $s_{n, m}^{d}[t]=1$ to represent that IUAV $n$ and RUAV $m$ form the first-hop link at time slot $t$, otherwise, $s_{n, m}^{d}[t]=0$. Similarly, $s_{k, k^{\prime}}^{p}=1$ represents that segment $l_{k, k^{\prime}}$ is chosen, and $s_{k, k^{\prime}}^{p}=$ 0 , otherwise. The set of optimization variables is defined as $\left\{\mathcal{V}_{K}, \mathcal{S}_{N}^{d}, \mathcal{S}_{K}^{p}, \mathcal{F}_{K}^{c}, \mathcal{P}_{N}^{d}\right\}$, where $\mathcal{V}_{K}=\left\{v_{k, k^{\prime}} \mid k, k^{\prime} \in \mathcal{K}, k \neq\right.$ $\left.k^{\prime}\right\}, \mathcal{S}_{N}^{d}=\left\{s_{n, m}^{d}[t] \mid n \in \mathcal{N}, m \in \mathcal{M}, t \in \mathcal{T}_{s}\right\}, \mathcal{S}_{K}^{p}=\left\{s_{k, k^{\prime}}^{p} \mid\right.$ $\left.k, k^{\prime} \in \mathcal{K}, k \neq k^{\prime}\right\}, \mathcal{F}_{K}^{c}=\left\{f_{k, k^{\prime}} \mid k, k^{\prime} \in \mathcal{K}, k \neq k^{\prime}\right\}$, and $\mathcal{P}_{N}^{d}=\left\{P_{n}^{d}[t] \mid n \in \mathcal{N}, t \in \mathcal{T}_{s}\right\}$.

The formulated energy-efficient trajectory scheduling, velocity control, frequency regulation, relay selection, and power allocation problem is given as follows:

$$
\begin{aligned}
& \text { P1 : } \min _{\left\{\mathcal{V}_{K}, \mathcal{S}_{N}^{d}, \mathcal{S}_{K}^{p}, \mathcal{F}_{K}^{c}, \mathcal{P}_{N}^{d}\right\}} \sum_{k \in \mathcal{K}} \sum_{k^{\prime} \neq k, k^{\prime} \in \mathcal{K}} s_{k, k^{\prime}}^{p}\left(N E_{k, k^{\prime}}^{p}\left(v_{k, k^{\prime}}\right)\right. \\
& +\sum_{n \in \mathcal{N}} \sum_{t \in \mathcal{T}_{s}} \sum_{m \in \mathcal{M}_{k, k^{\prime}}} s_{n, m}^{d}[t] E_{k, k^{\prime}}^{n, d}\left(P_{n}^{d}[t]\right) \\
& \left.+N E_{k, k^{\prime}}^{c}\left(f_{k, k^{\prime}}, v_{k, k^{\prime}}\right)\right) \\
& \text { s.t. } C_{1}: \frac{1}{f_{k, k^{\prime}}} \leq T_{s}, \forall f_{k, k^{\prime}} \in \mathcal{F}_{K}^{c} \text {, } \\
& C_{2}: \operatorname{Pr}\left(\delta_{k, k^{\prime}}\right)^{f_{k, k^{\prime}}} \leq P_{\max }, \forall f_{k, k^{\prime}} \in \mathcal{F}_{K}^{c}, \\
& C_{3}: v_{k, k^{\prime}, \min } \leq v_{k, k^{\prime}} \leq v_{k, k^{\prime}, \max }, \forall v_{k, k^{\prime}} \in \mathcal{V}_{K} \text {, } \\
& C_{4}: P_{n, \min } \leq P_{n}^{d}[t] \leq P_{n, \max }, \forall t \in \mathcal{T}_{s} \\
& C_{5}: \sum_{m \in \mathcal{M}_{k, k^{\prime}}} S_{n, m}^{d}[t] \leq 1, n \in \mathcal{N}, \forall t \in \mathcal{T}_{s}, \\
& C_{6}: \min \left(\gamma_{n, m}, \gamma_{m, B_{0}}, \gamma_{n, m, B_{0}}\right) \geq \gamma_{0}, \\
& \forall n \in \mathcal{N}, \forall m \in \mathcal{M}_{k, k^{\prime}} \text {. }
\end{aligned}
$$

Here, $C_{1}$ guarantees that the signal duration should be less than channel coherence time. $C_{2}$ denotes the minimum collision probability required for collision avoidance. $C_{3}$ and $C_{4}$ specify the IUAV velocity and transmission power constraints, respectively. $C_{5}$ ensures that at most one RUAV can be utilized as the relay station for IUAV at any time. $C_{6}$ denotes the 
quality of service (QoS) requirements defined in terms of minimum SNR threshold.

\section{Two-Stage Energy-efFicient Power Line INSPECTION ALGORITHM}

In this section, we introduce the energy-efficient power line inspection algorithm by combining DP, auction theory, and matching theory. Firstly, the NP-hard joint optimization problem is transformed to a two-stage optimization problem based on energy consumption magnitude and optimization timescale differences. Then, the large-timescale optimization problem is solved in the first stage based on DP. Next, the smalltimescale optimization problem is solved in the second stage by combining auction theory and matching theory. Finally, the analysis of theoretical properties is elaborated.

\section{A. Problem Transformation}

Problem $\mathbf{P 1}$ is formulated as a joint optimization problem, which involves both the large-timescale optimization such as trajectory scheduling, velocity control, and frequency regulation, the values of which are fixed during a segment and vary in the timescale of seconds, and the small-timescale optimization such as relay selection and power allocation, the values of which depend on channel fading and vary in the timescale of milliseconds.

It is infeasible to obtain the optimal solution in polynomial time due to the fact that the large-timescale and smalltimescale optimization variables of $\mathbf{P} \mathbf{1}$ are coupled with each other through the total number of time slots. Furthermore, the energy consumption objective value of the large-timescale optimization problem is generally several orders of magnitudes higher than that of the small-timescale optimization problem.

Therefore, to provide a solution, the timescale difference between the large-timescale optimization and the small-timescale optimization is utilized as a pre-knowledge to simplify the problem. Correspondingly, problem $\mathbf{P} \mathbf{1}$ can be transformed into a two-stage suboptimal problem, in which the largetimescale problem is solved in the first stage without considering the second stage optimization problem, and then the small-timescale problem is solved in the second stage based on the optimal results obtained in the first stage. Therefore, the proposed two-stage algorithm will lead to a suboptimal performance since trajectory scheduling, velocity control, frequency regulation, relay selection, and power allocation are not jointly optimized simultaneously. The first-stage optimization problem is given by

$$
\begin{array}{ll}
\text { P2 : } & \min _{\left\{\mathcal{V}_{K}, \mathcal{S}_{K}^{p}, \mathcal{F}_{K}^{c}\right\}} \sum_{k \in \mathcal{K}} \sum_{k^{\prime} \neq k, k^{\prime} \in \mathcal{K}} s_{k, k^{\prime}}^{p}\left(N E_{k, k^{\prime}}^{p}\left(v_{k, k^{\prime}}\right)\right. \\
& \left.+N E_{k, k^{\prime}}^{c}\left(f_{k, k^{\prime}}, v_{k, k^{\prime}}\right)\right) \\
\text { s.t. } & C_{1} \sim C_{3} .
\end{array}
$$

The second-stage optimization problem is given by

$$
\begin{array}{ll}
\text { P3 : } & \min _{\left\{\mathcal{S}_{N}^{d}, \mathcal{S}_{K}^{p}, \mathcal{P}_{N}^{d}\right\}} \sum_{k \in \mathcal{K}_{k^{\prime}} \neq k, k^{\prime} \in \mathcal{K}} \sum_{n \in \mathcal{N}} \sum_{t \in \mathcal{T}_{s}} \sum_{m \in \mathcal{M}_{k, k^{\prime}}} \\
& s_{k, k^{\prime}}^{p} s_{n, m}^{d}[t] E_{k, k^{\prime}}^{n, d}\left(\mathcal{P}_{n}^{d}[t]\right), \\
\text { s.t. } & C_{4} \sim C_{6} .
\end{array}
$$

In this fashion, the coupling between the large-timescale optimization and the small-timescale optimization variables disappears, and a two-stage low-complexity suboptimal algorithm can be developed by exploring DP, auction theory, and matching theory, which is elaborated in the next two subsections.

\section{B. DP-based Energy-efficient Trajectory Scheduling, Velocity Control, and Frequency Regulation}

The first-stage problem $\mathbf{P 2}$ has two principal features: 1) the decisions are made dynamically in discrete-time stages; 2 ) the energy consumption is additive over time (or stages), which is within the framework of the deterministic finite-state travel salesman problem (TSP), and can be subsequently solved by using DP.

TSP [27] is a typical NP-hard problem in which the computational complexity rises exponentially with the number of parameters. Several heuristic methods and software have been proposed to solve TSP for computational time reduction, e.g. Concorde [28], neural networks [29], Genetic algorithms (GAs) [30], ant colony optimization (ACO) algorithms [31], and GR-2opt [32].

However, conventional heuristic algorithms which are designed for minimizing the total traveling distance in TSP problems cannot be directly applied here since the optimization objective here is to minimize the total energy consumption. The calculation of the energy consumed for flying through each sement involves the joint optimization of trajectory scheduling, velocity control, and frequency regulation, which cannot be directly solved by existing heuristic algorithms. In this work, problem P2 is solved subsequently by the DP algorithm based on the obtained minimum energy consumption of each segment. The reason lying behind is that DP is a classic algorithm to obtain the optimal solution, which can serve as an upper performance benchmark for evaluating other heuristic algorithms.

In DP, the evolution of the system state under the influence of decisions taken at discrete stages is given by $x_{\tau+1}=$ $z\left(x_{\tau}, u_{\tau}\right), \tau=0,1,2, \cdots, \psi-1$, where $\psi$ is the total number of stages, $\tau$ is the stage index, $x_{\tau}$ and $x_{\tau+1}$ are the states of stage $\tau$ and stage $\tau+1$, respectively, $u_{\tau}$ is the decision made at stage $\tau$, and $z$ enumerates the updating mechanism of state. By setting the set of states as the set of target points, e.g., $x_{\tau}=k$, and the set of decisions as the set of trajectory scheduling variables, e.g., $u_{\tau}=s_{k, k^{\prime}}^{p}[\tau]$, the state updating form is given by

$$
\left.x_{\tau+1}\right|_{x_{\tau}=k}=\sum_{k^{\prime} \neq k, k^{\prime} \in \mathcal{K}} k^{\prime} s_{k, k^{\prime}}^{p}[\tau]
$$


The cost function in stage $\tau$, i.e., $g\left(x_{\tau}, u_{\tau}\right)$, is additive over stage, which is given by

$$
\left.g\left(x_{\tau}, u_{\tau}\right)\right|_{\left(x_{\tau}=k, u_{\tau}=s_{k, k^{\prime}}^{p}[\tau]\right)}=\sum_{k^{\prime} \neq k, k^{\prime} \in \mathcal{K}} s_{k, k^{\prime}}^{p}[\tau] E_{k, k^{\prime}}^{*}
$$

Here, $E_{k, k^{\prime}}^{*}$ denotes the minimum energy consumption required to move from target point $k$ to target point $k^{\prime}$. For the deterministic finite-state TSP where $\psi$ is finite, the minimum energy consumption of IUAV $n$ with the initial stage $x_{0}=0$ $(\tau=0, k=0)$ is given by

$$
J^{*}\left(x_{0}\right)=\min _{\mathcal{S}_{K}^{p}}\left[\sum_{\tau=0}^{\psi-1} g\left(x_{\tau}, u_{\tau}\right)+g(\psi)\right],
$$

where $g(\psi)$ is the terminal cost which represents the minimum energy consumption of the last segment. $J^{*}\left(x_{0}\right)$ can be obtained by using DP, which proceeds backward from stage $\psi-1$ to stage 0 as

$$
\begin{gathered}
J_{\psi}\left(x_{\psi}\right)=g\left(x_{\psi}\right), \\
J_{\tau}\left(x_{\tau}\right)=\min _{\substack{s_{k, k^{\prime}}^{p}\\
}}\left\{g\left(x_{\tau}, u_{\tau}\right)+J_{\tau+1}\left(z\left(x_{\tau}, u_{\tau}\right)\right)\right\}, \\
\tau=0,1, \cdots, \psi-1 .
\end{gathered}
$$

However, the above DP-based trajectory scheduling algorithm involves a set of unknown parameters, i.e., $\left\{E_{k, k^{\prime}}^{*} \mid\right.$ $\left.k, k^{\prime} \in \mathcal{K}, k^{\prime} \neq k\right\}$, which can be obtained by solving the following joint velocity and frequency regulation problem

$$
\begin{aligned}
& \text { P4: } \min _{\left\{\mathcal{V}_{k}, \mathcal{F}_{N}^{c}\right\}} d_{k, k^{\prime}}\left(\frac{c_{k, k^{\prime}}^{1}\left(v_{k, k^{\prime}}\right)^{4}+c_{k, k^{\prime}}^{2}}{\left(v_{k, k^{\prime}}\right)^{2}}+\frac{P^{c} f_{k, k^{\prime} t_{c}}}{v_{k, k^{\prime}}}\right), \\
& \text { s.t. } \quad C_{1} \sim C_{3} .
\end{aligned}
$$

Considering the $f_{k, k^{\prime}}$ related constraints $C_{1}$ and $C_{2}$ in $\mathbf{P} \mathbf{4}$, we have

$$
f_{k, k^{\prime}}=\max \left(\frac{v_{k, k^{\prime}}}{c_{3}}, \frac{\log _{2} P_{\max }}{\log _{2} P_{r}\left(\delta_{k, k^{\prime}}\right)}\right) .
$$

$\mathbf{P} 4$ can be further divided into two scenarios based on velocity range, i.e., the high-velocity scenario and the low-velocity scenario.

In the high-velocity scenario, i.e., $\frac{v_{k, k^{\prime}}}{c_{3}} \geq \frac{\log _{2} P_{\max }}{\log _{2} P_{r}\left(\delta_{k, k^{\prime}}\right)}$, we have $f_{k, k^{\prime}}=\frac{v_{k, k^{\prime}}}{c_{3}}$, and $\mathbf{P} 4$ is written as

$$
\begin{aligned}
& \text { P5 : } \min _{v_{k, k^{\prime}}} d_{k, k^{\prime}}\left(\frac{c_{k, k^{\prime}}^{1}\left(v_{k, k^{\prime}}\right)^{4}+c_{k, k^{\prime}}^{2}}{\left(v_{k, k^{\prime}}\right)^{2}}+\frac{P^{c} t_{c}}{c_{3}}\right), \\
& \text { s.t. } \quad C_{1} \sim C_{3} .
\end{aligned}
$$

In the low-velocity scenario, i.e., $\frac{\log _{2} P_{\max }}{\log _{2} P_{r}\left(\delta_{k, k^{\prime}}\right)}>\frac{v_{k, k^{\prime}}}{c_{3}}$, we have $f_{k, k^{\prime}}=\frac{\log _{2} P_{\max }}{\log _{2} P_{r}\left(\delta_{k, k^{\prime}}\right)}$, and $\mathbf{P} \mathbf{4}$ is written as

P6 : $\min _{v_{k, k^{\prime}}} d_{k, k^{\prime}}\left(\frac{c_{k, k^{\prime}}^{1}\left(v_{k, k^{\prime}}\right)^{4}+c_{k, k^{\prime}}^{2}}{\left(v_{k, k^{\prime}}\right)^{2}}+\frac{P^{c} t_{c} \log _{2} P_{\max }}{v_{k, k^{\prime}} \log _{2} P_{r}\left(\delta_{k, k^{\prime}}\right)}\right)$, s.t. $\quad C_{1} \sim C_{3}$.

It is noted that both P5 and P6 are convex, and can be easily solved by using the KKT conditions. Defining $\hat{v}_{k, k^{\prime}}^{*}$ and $\tilde{v}_{k, k^{\prime}}^{*}$ as the optimal solutions of $\mathbf{P 5}$ and $\mathbf{P 6}$, respectively, the optimal velocity is determined as

$$
\begin{aligned}
v_{k, k^{\prime}}^{*}= & \underset{\substack{\left\{\hat{v}_{k, k^{\prime}}^{*} \tilde{v}_{k, k^{\prime}}^{*}\right\} \\
\arg \min }}{ }\left(\left\{E_{k, k^{\prime}}\left(\hat{v}_{k, k^{\prime}}^{*}, \frac{\hat{v}_{k, k^{\prime}}^{*}}{c_{3}}\right),\right.\right. \\
& \left.\left.E_{k, k^{\prime}}\left(\tilde{v}_{k, k^{\prime}}^{*}, \frac{\log _{2} P_{\max }}{\log _{2} P_{r}\left(\delta_{k, k^{\prime}}\right)}\right)\right\}\right) .
\end{aligned}
$$
(19).

Then, the optimal frequency can be determined by $v_{k, k^{\prime}}^{*}$ as

\section{Auction-matching based Energy-efficient Relay Selection and Power Allocation}

Based on the optimal flying trajectory and velocity obtained in the first stage, the total number of time slots in segment $l_{k, k^{\prime}}$ can be determined. Assuming that $M_{k, k^{\prime}}$ RUAVs are available when IUAVs are flying through $l_{k, k^{\prime}}$, the second-stage energyefficient relay selection and power allocation problem given in P3 becomes a two-sided matching problem with $N$ IUAVs on one side, and $M_{k, k^{\prime}}$ RUAVs on the other side.

The two-sided matching is denoted as $\chi$, which maps elements in set $\mathcal{N}$ to set $\mathcal{M}_{k, k^{\prime}}$ in a one-to-one fashion. For an instance, $\chi(n)=m$ represents that IUAV $n$ is matched with RUAV $m$. In general, $\chi$ is obtained based on the preference, which is defined as the reciprocal of the minimum achievable energy consumption minus the matching cost. The cost set of RUAVs is defined as $\Lambda=\left\{\xi_{1}, \cdots, \xi_{m}, \cdots, \xi_{M_{k, k^{\prime}}}\right\}$, i.e., the cost of selecting RUAV $m$ for any IUAV is $\xi_{m}$. Hence, the preference value of IUAV $n$ towards RUAV $m$ is calculated as

$$
\hat{E}_{k, k^{\prime}}^{n, m}=\frac{1}{E_{k, k^{\prime}}^{n, d}\left(P_{n}^{d *}[t]\right)}-\xi_{m},
$$

where $P_{n}^{d *}[t]$ is the optimal power allocation strategy, which can be obtained by solving the following power allocation problem

$$
\begin{aligned}
& \text { P7 : } \min _{\mathcal{P}_{n}^{d}[t]} E_{k, k^{\prime}}^{n, d}\left(P_{n}^{d}[t]\right), \\
& \text { s.t. } C_{4}, C_{6} .
\end{aligned}
$$

By solving (24), the optimal power allocation strategy is given by

$$
P_{n}^{d *}[t]=\min \left(\max \left(P_{n}^{d}[t], P_{n, \min }\right), P_{n, \max }\right),
$$

where

$$
P_{n}^{d}[t]=\frac{\gamma_{0} g_{m, B_{m}} N_{0} P_{m}^{d}[t]+\gamma_{0} N_{0}^{2}}{g_{n, m} g_{m, B_{0}} P_{m}^{d}[t]-\gamma_{0} g_{n, m} N_{0}} .
$$

Then, the preference list of any IUAV $n$, i.e., $\Phi_{n}$, is established by sorting $M$ RUAVs in a descending order according to $\hat{E}_{k, k^{\prime}}^{n, m}$.

During the iterative matching process, a matching request is sent from any IUAV that remains unmatched to its most preferred RUAV in the preference list. If there exists only 
one matching request, then a stable matching is formed accordingly. A matching contention arises when multiple IUAVs simultaneously send matching requests to the same RUAV. We propose an English auction strategy to resolve the contention and update preference.

English auction can be regarded as a typical second-price auction, in which the winner has to outbid the second-highest bidder with a predefined minimum increment. The auction model is composed of four fundamental elements: 1) Auction goods: the set of RUAVs that are requested by multiple IUAVs, which is defined as $\mathcal{G}$; 2) Auctioneer: the macro BS which determines the allocation of $\mathcal{G}$ based on the principle of auction; 3) Bidders: the set of IUAVs that request the same RUAV, which is defined as $\mathcal{B}$; 4) Bidding Price: the cost set $\Lambda$.

For any RUAV in $\mathcal{G}$, an auction is implemented as follows.

In the $i$-th bidding round, the bidding price of bidders, i.e., IUAVs in $\mathcal{B}$, is increased by a predefined amount $\Delta \xi_{m}$, which is given by

$$
\xi_{m}[i]=\xi_{m}[i-1]+\Delta \xi_{m} .
$$

Then, the preference lists of bidders are also updated based on the latest bidding price, since the preference of any bidder towards RUAV $m$ is decreased by an amount $\Delta \xi_{m}$. As the bidding iteration proceeds, any bidder except the winner will quit the bidding due to the continuous growth of the matching cost, i.e., some other URAV $m^{\prime}\left(m^{\prime} \neq m\right)$ is more preferred than RUAV $m,\left.\hat{E}_{k, k^{\prime}}^{n, d}\right|_{\chi(n)=m}<\left.\hat{E}_{k, k^{\prime}}^{n, d}\right|_{\chi(n)=m^{\prime}}$. The auction terminates when no bidder is willing to outbid the current price, and RUAV $m$ is matched with the IUAV who bids the highest price.

The matching process will continue until either any IUAV has been matched with some RUAVs, or none RUAV is available for relaying.

\section{Property Analysis}

In this subsection, we provide the convergence and complexity analysis.

By employing the concept from the matching theory [33], the following convergence and stability properties can be derived. The proof is omitted here due to space limitation.

Theorem 1: $\chi$ generated by the auction-matching based joint relay selection and power allocation algorithm converges to a stable matching.

Based on [34], the computational complexity of the DPbased energy-efficient trajectory scheduling, velocity control, and frequency regulation algorithm is $\mathcal{O}\left(\psi^{2} 2^{\psi-1}\right)$. For the auction-matching based relay selection and power allocation algorithm, the corresponding computational complexity is $\mathcal{O}\left(N N^{\text {loop }}\right)$ if $N \geq M_{k, k^{\prime}}$, or $\mathcal{O}\left(M_{k, k^{\prime}} N^{\text {loop }}\right)$ if $M_{k, k^{\prime}} \geq N$, where $N^{\text {loop }}$ is the number of bidding rounds.

\section{Vi. Performance Evaluation}

In this section, the proposed two-stage energy consumption minimization algorithm is evaluated based on real-world map and realistic power grid topology, which is shown in Fig. 2. The solar farm located in Newton County, Mississippi, is

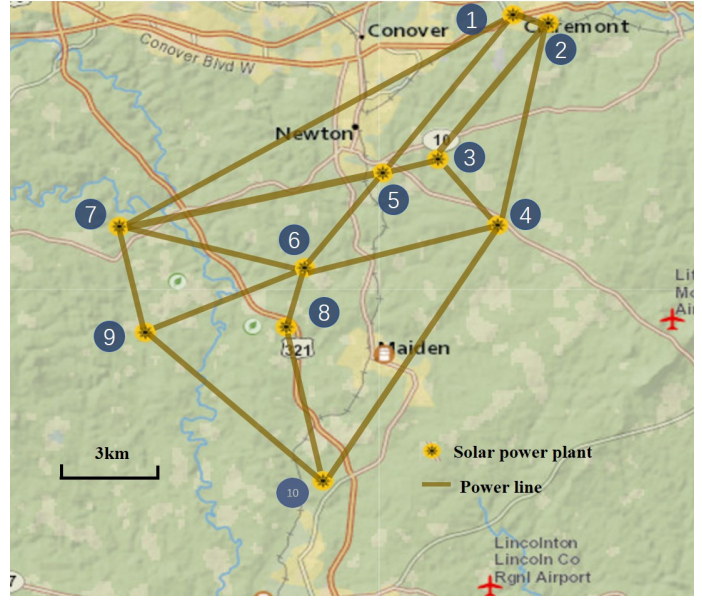

Fig. 2: A snapshot of the solar farm located in Newton County, Mississippi.

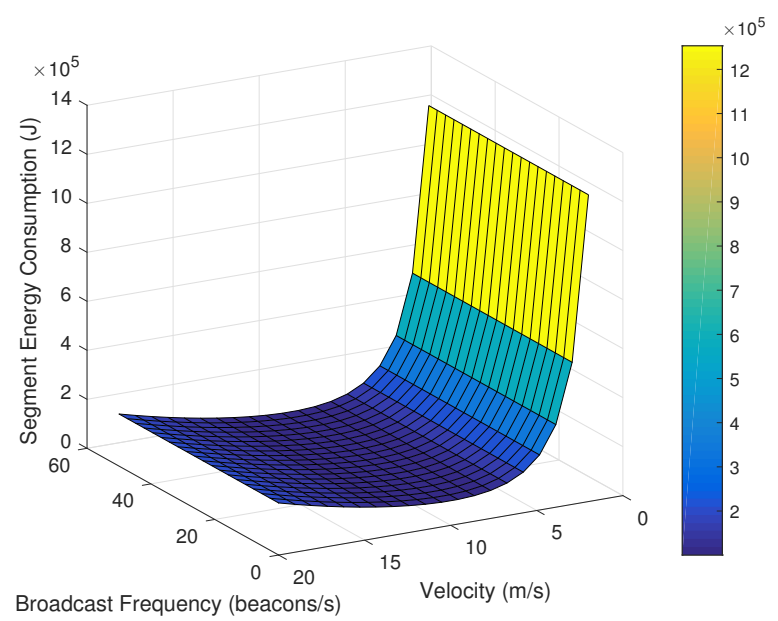

Fig. 3: The relationship among segment energy consumption, flight velocity, and beacon broadcasting frequency.

selected as the simulation scenario, where solar power plants are represented by small yellow points. The corresponding geographical and capacity data are obtained from both U.S. Energy Information Administration (EIA) [35] and Google Earth. An example of plant information is shown in Table I. Simulation parameters are shown in Table II.

Fig. 3 shows the relationship among segment energy consumption (e.g., $E_{k, k^{\prime}}$ ), flight velocity, and broadcasting frequency of distance estimation beacons. It is noted that the energy consumption decreases firstly and then increases with velocity, while increases monotonically with broadcasting frequency. The reason is that whenever the increased propulsion energy consumption due to unit velocity increment is less than the amount of energy saved from flight duration reduction, the energy consumption decreases as velocity increases. Otherwise, the energy consumption increases with velocity.

Fig. 4 shows the impact of target point numbers on the total energy consumption. The greedy algorithm which always selects the segment with minimum energy consumption at 


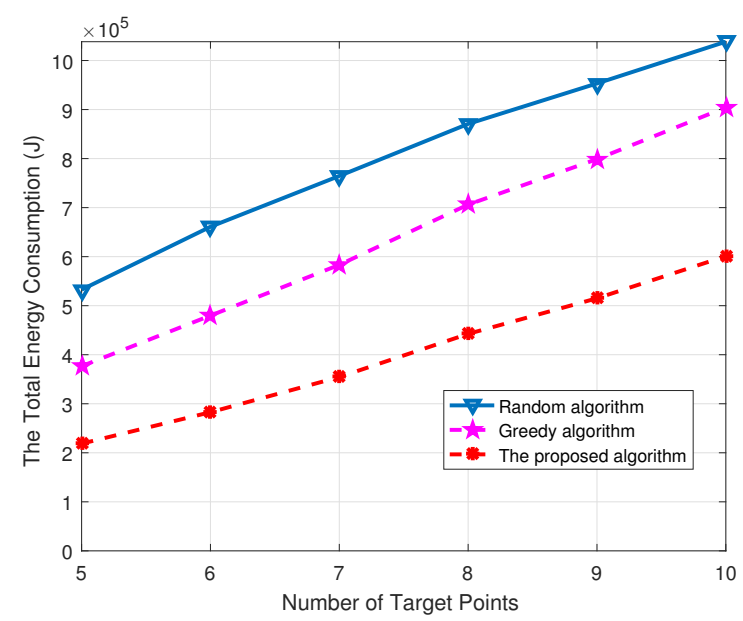

Fig. 4: The impact of target point numbers on the total energy consumption.

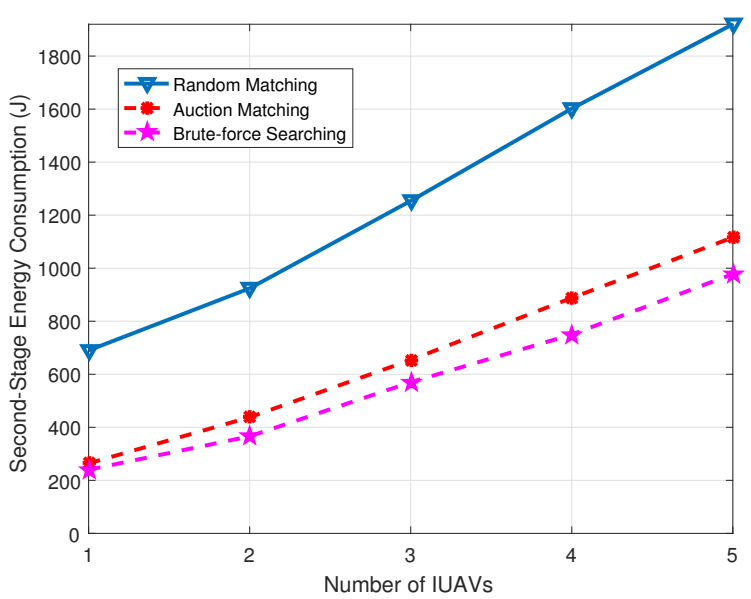

Fig. 5: The second-stage energy consumption versus the number of IUAVs.

each stage, and the random scheme which randomly determines trajectory, velocity, broadcasting frequency, relay, and transmission power, are used for comparison. Numerical results demonstrate that the energy consumption performances of all of the three algorithms deteriorate as the number of target points increases. Nevertheless, the energy consumption increasing rate of the proposed algorithm, i.e., the curve slope, is much lower compared with the two heuristic algorithms due to the joint optimization of multiple variables from an energy efficiency perspective.

Fig. 5 shows the second-stage energy consumption versus the number of IUAVs. The brute-force searching scheme which achieves the optimum performance by examining every possible combination is used to served as the upper performance benchmark. It is shown that the proposed algorithm can achieve up to $87.6 \%$ of the optimum performance, and outperform the random allocation scheme by more than $41.8 \%$ when $N=5$. The reason lies behind is that relay selection

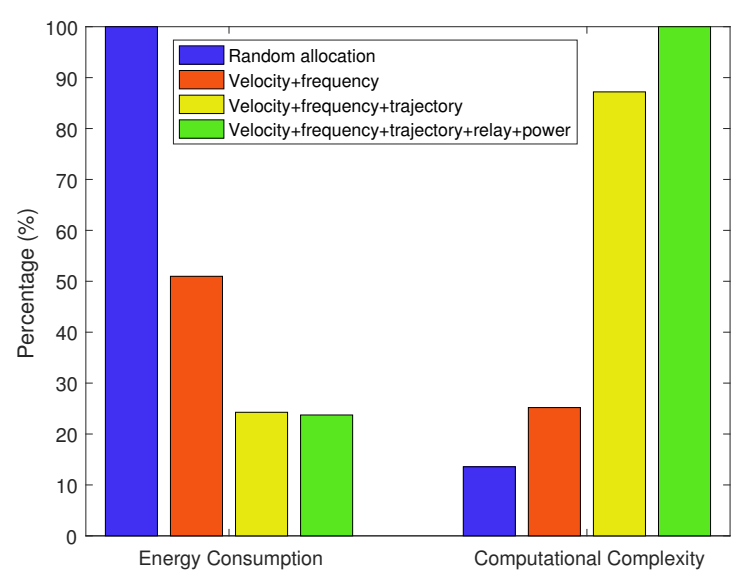

Fig. 6: The tradeoff between energy consumption reduction and computational complexity.

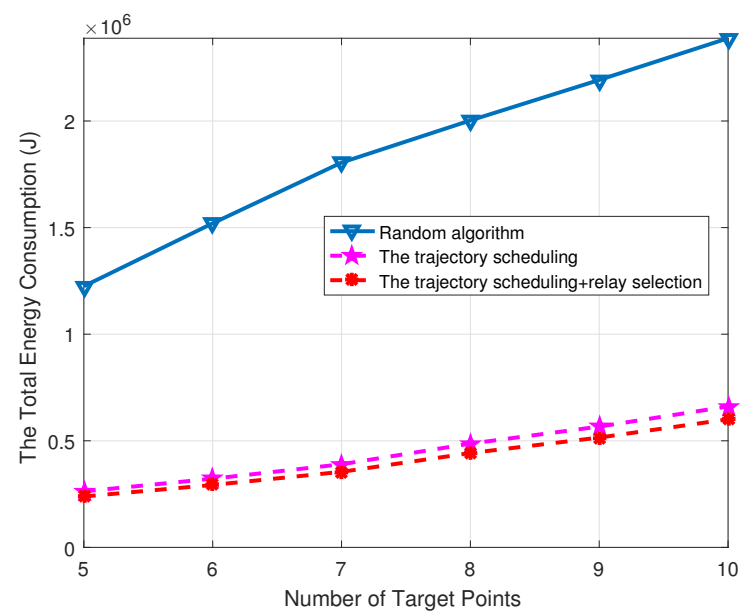

Fig. 7: The impact of trajectory scheduling optimization and relay selection optimization on the total energy consumption.

is not jointly optimized with power control in the heuristic algorithm.

Fig. 6 shows the tradeoff between energy consumption reduction and computational complexity. Numerical results demonstrate that the joint optimization of trajectory scheduling, velocity control, and frequency regulation could bring an energy saving gain up to $75.1 \%$ at the cost of a $73.2 \%$ computational complexity increment. In particular, it is noted that the energy saved from the joint optimization of relay selection and power allocation is trivial compared to the total amount of energy consumption. The reason lies behind is that the propulsion power is usually several orders of magnitudes higher than the data transmission power. Thus, proper consideration of energy saving gains and computational complexity costs are required to achieve satisfactory energy efficiency performance. This phenomenon is further revealed in Fig. 7, which demonstrates the energy saving could achieve $75.1 \%$ when the number of target points is 10 . 
TABLE I: An example of plant information

\begin{tabular}{|l|l|l|l|l|}
\hline Number & Plant Code & Capacity & Longitude & Latitude \\
\hline 1 & 59474 & $17.5 \mathrm{MW}$ & $35^{\circ} 42^{\prime} 54.8^{\prime \prime} \mathrm{N}$ & $81^{\circ} 09^{\prime} 01.0^{\prime \prime} \mathrm{W}$ \\
\hline 2 & 59519 & $3.5 \mathrm{MW}$ & $35^{\circ} 42^{\prime} 43.8^{\prime \prime} \mathrm{N}$ & $81^{\circ} 08^{\prime} 04.9^{\prime \prime} \mathrm{W}$ \\
\hline 3 & 58829 & $5 \mathrm{MW}$ & $35^{\circ} 39^{\prime} 21.7^{\prime \prime} \mathrm{N}$ & $81^{\circ} 11^{\prime} 05.9^{\prime \prime} \mathrm{W}$ \\
\hline
\end{tabular}

TABLE II: Simulation Parameters.

\begin{tabular}{|l|l|}
\hline Simulation Parameters & Value \\
\hline Propulsion power related coefficient $c_{k, k^{\prime}}^{1}$ & $0.001 \sim 0.1$ \\
\hline Propulsion power related coefficient $c_{k, k^{\prime}}^{2}$ & $20 \sim 2000$ \\
\hline Channel coherence time related coefficient $c_{3}$ & $2.539 * 10^{-2}$ \\
\hline Beacon signal duration $t_{c}$ & $100 \mu \mathrm{s}$ \\
\hline Noise power $N_{0}$ & $-114 \mathrm{dbm}$ \\
\hline Collision probability threshold $P_{\max }$ & $10^{-4}$ \\
\hline SNR threshold $\gamma_{0}$ & $20 \mathrm{db}$ \\
\hline Maximum transmission power for IUAV $n P_{n, \max }$ & $20 \mathrm{dbm}$ \\
\hline Minimum transmission power fot IUAV $n P_{n, \min }$ & $0 \mathrm{dbm}$ \\
\hline Maximum flight velocity $V_{k, k^{\prime}, \max }$ & $20 \mathrm{~m} / \mathrm{s}$ \\
\hline Minimum flight velocity $V_{k, k^{\prime}, \min }$ & $2 \mathrm{~m} / \mathrm{s}$ \\
\hline Number of IUAV & $1 \sim 5$ \\
\hline Number of RUAV & 6 \\
\hline Estimations errors $\delta_{k, k^{\prime}}$ & $2 \sim 4 \mathrm{~m}$ \\
\hline The probability of estimations errors $\operatorname{Pr}\left(\delta_{k, k^{\prime}}\right)$ & $0.1 \sim 0.2$ \\
\hline Number of target points $K$ & 10 \\
\hline Cell radius of base station & $5 \mathrm{~km}$ \\
\hline
\end{tabular}

\section{CONCLUSION}

In this paper, we investigated IIoUAVs based power line inspection in smart grid, and proposed a two-stage energyefficient joint trajectory scheduling, velocity control, frequency regulation, relay selection, and power allocation algorithm by combining DP, auction theory, and matching theory. The proposed algorithm was compared with other heuristic algorithms based on real-world map and realistic power grid topology, and its superior performance in energy consumption reduction was validated through numerical results. In future works, we will focus on how to jointly optimize trajectory scheduling and collision avoidance via machine learning based approaches.

\section{REFERENCES}

[1] A. Golshani, W. Sun, Q. Zhou, Q. P. Zheng, and J. Tong, "Two-stage adaptive restoration decision support system for a self-healing power grid," IEEE Trans. Ind. Informat., vol. PP, no. 99, pp. 1-1, Jun. 2017.

[2] Y. Zhang, R. Yu, M. Nekovee, Y. Liu, S. Xie, and S. Gjessing, "Cognitive machine-to-machine communications: Visions and potentials for the smart grid," IEEE Netw. Mag., vol. 26, no. 3, pp. 6-13, May. 2012.

[3] S. Maharjan, Q. Zhu, Y. Zhang, S. Gjessing, and T. Basar, "Dependable demand response management in the smart grid: A stackelberg game approach," IEEE Trans. Smart Grid, vol. 4, no. 1, pp. 120-132, Mar. 2013.

[4] A. M. Prostejovsky, O. Gehrke, A. M. Kosek, T. Strasser, and H. W. Bindner, "Distribution line parameter estimation under consideration of measurement tolerances," IEEE Trans. Ind. Informat., vol. 12, no. 2, pp. 726-735, Feb. 2016.

[5] B. Xiao and S. Yin, "A new disturbance attenuation control scheme for quadrotor unmanned aerial vehicles," IEEE Trans. Ind. Informat., vol. PP, no. 99, pp. 1-1, Mar. 2017.

[6] J. Cui, Y. Zhang, S. Ma, Y. Yi, J. Xin, and D. Liu, "Path planning algorithms for power transmission line inspection using unmanned aerial vehicles," in Proc. IEEE CCDC '17, Chongqing, China, May. 2017, pp. 2304-2309.
[7] N. H. Motlagh, T. Taleb, and O. Arouk, "Low-altitude unmanned aerial vehicles-based internet of things services: Comprehensive survey and future perspectives," IEEE Internet Things J., vol. 3, no. 6, pp. 899922, Sep. 2016.

[8] Y. Zhang, R. Yu, W. Yao, S. Xie, Y. Xiao, and M. Guizani, "Home M2M networks: Architectures, standards, and QoS improvement," IEEE Commun. Mag., vol. 49, no. 4, pp. 44-52, Apr. 2011.

[9] Z. Zhou, J. Gong, Y. He, and Y. Zhang, "Software defined machine-tomachine communication for smart energy management," IEEE Commun. Mag., vol. 55, no. 10, pp. 52-60, Oct. 2017.

[10] L. Gupta, R. Jain, and G. Vaszkun, "Survey of important issues in UAV communication networks," IEEE Commun. Surv. Tut., vol. 18, no. 2, pp. 1123-1152, Nov. 2016.

[11] D. Choi, S. Kim, and D. Sung, "Energy-efficient maneuvering and communication of a single UAV-Based relay," IEEE Trans. Aerosp. Electron. Syst., vol. 50, no. 3, pp. 2320-2327, Dec. 2014.

[12] H. Lee, H. Yoo, and B. Lee, "Deployment method of UAVs with energy constraint for multiple tasks," IEEE Electron. Lett., vol. 51, no. 21, pp. 1650-1652, Oct. 2015.

[13] W. Benitez, Y. Bogado, A. Guerrero, and M. Arzamendia, "Development of an UAV prototype for visual inspection of aerial electrical lines," in Proc. IEEE CASE '16, Buenos Aires, Argentina, Aug. 2016, pp. 7-12.

[14] Z. Guan, J. Li, L. Zhu, X. Du, and M. Guizani, "Toward delaytolerant flexible data access control for smart grid with renewable energy resources," IEEE Trans. Ind. Informat., vol. 13, no. 6, pp. 3216-3225, Dec. 2017.

[15] T. Santos, M. Moreira, J. Almeida, A. Dias, A. Martins, J. Dinis, J. F. ormiga, and E. Silva, "PLineD: Vision-based power lines detection for unmanned aerial vehicles," in Proc. IEEE ICARSC '17, Coimbra, Portugal, Apr. 2017, pp. 253-359.

[16] V. Roberge, M. Tarbouchi, and G. Labonte, "Comparison of parallel genetic algorithm and particle swarm optimization for real-time UAV path planning," IEEE Trans. Ind. Informat., vol. 9, no. 1, pp. 132-141, May. 2013.

[17] Y. Zeng and R. Zhang, "Energy-efficient UAV communication with trajectory optimization," IEEE Trans. Wirel. Commun., vol. 16, no. 6, pp. 3747-3760, Mar. 2017.

[18] M. Mozaffari, W. Saad, M. Bennis, and M. Debbah, "Mobile unmanned aerial vehicles (UAVs) for energy-efficient internet of things communications," IEEE Trans. Wirel. Commun., vol. PP, no. 99, pp. 1-1, Sep. 2017.

[19] E. Bertran and A. Cerda, "On the tradeoff between electrical power consumption and flight performance in fixed-wing UAV autopilots," IEEE Trans. Veh. Technol., vol. 65, no. 11, pp. 8832-8840, Aug. 2016.

[20] J. Lee and K. Yu, "Optimal path planning of solar-powered UAV using gravitational potential energy," IEEE Trans. Aerosp. Electron. Syst., vol. 53, no. 3, pp. 1442-1451, Feb. 2017.

[21] C. Luo, S. McClean, G. Parr, L. Teacy, and R. D. Nardi, "UAV position estimation and collision avoidance using the extended Kalman filter," IEEE Trans. Veh. Technol., vol. 62, no. 6, pp. 2749-2762, Jan. 2013.

[22] Y. Fu, Y. Zhang, and X. Yu, "An advanced sense and collision avoidance strategy for unmanned aerial vehicles in landing phase," IEEE Aerosp. Electron. Syst. Mag., vol. 31, no. 9, pp. 40-52, Oct. 2016.

[23] V. Vahidi, A. P. Yazdanpanah, E. Saberinia, and E. E. Regentova, "Channel estimation, equalisation, and evaluation for high-mobility airborne hyperspectral data transmission," IET Commun., vol. 10, no. 18, pp. 2656-2662, Dec. 2016.

[24] M. C. Mah, H. S. Lim, and A. W. C. Tan, "Improved channel estimation for MIMO interference cancellation," IEEE Commun. Lett., vol. 19, no. 8, pp. 1355-1357, Jun. 2015.

[25] C. Xu, J. Feng, B. Huang, Z. Zhou, S. Mumtaz, and J. Rodriguez, "Joint relay selection and resource allocation for energy-efficient D2D cooperative communications using matching theory," Appl. Sci., vol. 7, no. 5, pp. 1-24, 2017.

[26] T. Kim and M. Dong, "An iterative Hungarian method to joint relay selection and resource allocation for D2D communications," IEEE Wirel. Commun. Lett., vol. 3, no. 6, pp. 625-628, Jul. 2014.

[27] R. Baraglia, J. I. Hidalgo, and R. Perego, "A hybrid heuristic for the traveling salesman problem," IEEE Tran. Evol. Comput., vol. 5, no. 6, pp. 613-622, Dec. 2001.

[28] W. J. Cook, In pursuit of the traveling salesman: mathematics at the limit of computation. Princeton, NJ, USA: Princeton Univ. Press, 2011.

[29] M. S. Tarzjan, M. Khademi, M. Akbarzadeh-T, and H. A. Moghaddam, "A novel constructive-optimizer neural network for the traveling salesman problem," IEEE Tran. Syst., Man, Cybern., Syst., vol. 37, no. 4, pp. 754-770, Jul. 2007. 
[30] H. D. Nguyen, I. Yoshihara, K. Yamamori, and M. Yasunaga, "Implementation of an effective hybrid GA for large-scale traveling salesman problems," IEEE Tran. Syst., Man, Cybern., Syst., vol. 37, no. 1, pp. 92-99, Jan. 2007.

[31] F. M. Mller and S. Yang, "Ant colony optimization with local search for dynamic traveling salesman problems," IEEE Tran. Cybern., vol. 47, no. 7, pp. 1743-1756, Jun. 2016.

[32] C. Tsai and Y. Chiang, "Enhancement of traveling salesman problem by hybridization of greedy and 2-opt techniques," in Proc. IEEE ICMLC '16, Jeju, South Korea, Jul. 2016, pp. 564-569.

[33] Z. Zhou, C. G. C. Xu, S. Mumtaz, and J. Rodriguez, "Social big data based content dissemination in internet of vehicles," IEEE Trans. Ind. Informat., vol. PP, no. 99, pp. 1-1, Jul. 2017.

[34] R. Bellman, "Dynamic programming treatment of the travelling salesman problem," J. Acm, vol. 9, no. 1, pp. 61-63, Nov. 1962.

[35] [Online]. Available: https://www.eia.gov/state/maps.php?src=home-f3

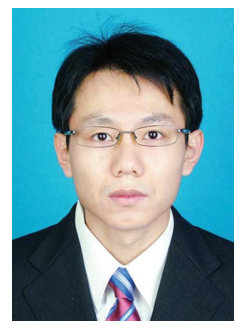

Zhenyu Zhou (M'11-SM'17) received his M.E. and $\mathrm{Ph} . \mathrm{D}$ degree from Waseda University, Tokyo, Japan in 2008 and 2011 respectively. From April 2012 to March 2013, he was the chief researcher at Department of Technology, KDDI, Tokyo, Japan. From March 2013 to now, he is an Associate Professor at School of Electrical and Electronic Engineering, North China Electric Power University, China. He is also a visiting scholar with Tsinghua-Hitachi Joint Lab on Environment-Harmonious ICT at University of Tsinghua, Beijing from 2014 to now. He served as an Associate Editor for IEEE Access, and a Guest Editor for IEEE Communications Magazine and Transactions on Emerging Telecommunications Technologies. He also served as workshop co-chair for IEEE ISADS 2015 , and TPC member for IEEE Globecom, IEEE CCNC, IEEE ICC, IEEE APCC, IEEE VTC, IEEE Africon, etc. He is a voting member of P1932.1 Working Group. He was the recipient of the IEEE Vehicular Technology Society "Young Researcher Encouragement Award" in 2009, the Beijing Outstanding Young Talent Award in 2016, the IET Premium Award in 2017, and the IEEE ComSoc Green Communications and Computing Technical Committee 2017 Best Paper Award. His research interests include green communications, vehicular communications, and smart grid communications. He is a senior member of IEEE.

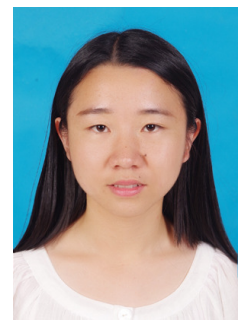

Chuntian Zhang is currently working towards the M.S. degree at North China Electric Power University, China. Her research interests include green communications and smart grid.

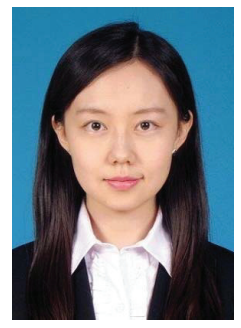

Chen Xu (S'12-M'15) received the B.S. degree from Beijing University of Posts and Telecommunications, in 2010, and the Ph.D. degree from Peking University, in 2015. She is now a lecturer in School of Electrical and Electronic Engineering, North China Electric Power University, China. Her research interests mainly include wireless resource allocation and management, game theory, optimization theory, heterogeneous networks, and smart grid communication. She received the best paper award in International Conference on Wireless Communications and Signal Processing (WCSP 2012), and she is the winner of IEEE Leonard G. Abraham Prize 2016.

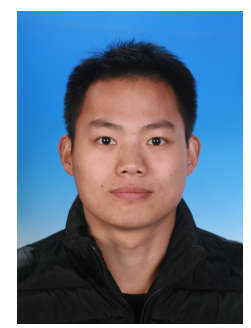

Fei Xiong is currently working towards the M.S. degree at North China Electric Power University, China. His research interests include green communications and smart grid.

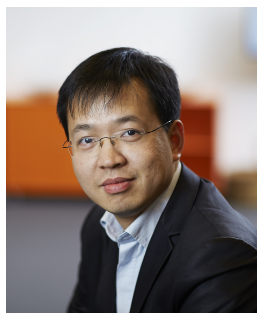

Yan Zhang (M'05-SM'10) received the Ph.D. degree from the School of Electrical and Electronics Engineering, Nanyang Technological University, Singapore. He is currently a Full Professor with the Department of Informatics, University of Oslo, Oslo, Norway. His current research interests include next-generation wireless networks leading to $5 \mathrm{G}$, green and secure cyber-physical systems, such as smart grid, healthcare, and transport. He is an IEEE Vehicular Technology Society (VTS) Distinguished Lecturer. He is also a Senior Member of the IEEE ComSoc, the IEEE CS, the IEEE PES, and the IEEE VTS. He is a fellow of the IET. He is an Associate Technical Editor of the IEEE Communications Magazine, an Editor of IEEE Network Magazine, an Editor of the IEEE Transactions on Green Communications and Networking, an Editor of the IEEE Communications Surveys and Tutorials, an Editor of IEEE Internet of Things Journal, and an Associate Editor of the IEEE Access. He serves as the Chair in a number of conferences, including the IEEE GLOBECOM 2017, the IEEE PIMRC 2016, the IEEE CloudCom 2016, the IEEE ICCC 2016, the IEEE CCNC 2016, the WCSP 2016, the IEEE SmartGridComm 2015, and the IEEE CloudCom 2015. He serves as a TPC member for numerous international conferences, including the IEEE INFOCOM, the IEEE ICC, the IEEE GLOBECOM, and the IEEE WCNC.

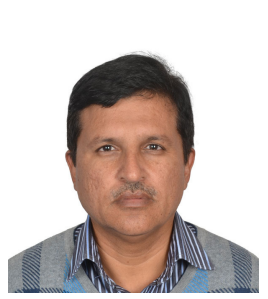

Tariq Umer (M'16-SM'16) received his Ph.D. in Communication systems in 2012 from School of Computing \& Communications, Lancaster University, U.K and Masters in Computer Science in 1997 from Bahauudin Zakariya University, Multan, Pakistan. He has served the IT education sector in Pakistan for more than 13 years. Since January 2007, he is working as Assistant Professor in CS department of COMSATS Institute of Information Technology, Wah Cantt. His research interests include cognitive radio ad hoc networks, Internet of Things, Wireless Sensor Networks, Telecommunication Network Design, Vehicular Adhoc Networks and Internet of Vehicles (IoV). He is currently serving as an Editorial board member of Elsevier Future Generation Computer System (FGCS) and Associate Editor of the IEEE Access journal. He served in the TPC for the international conference FIT 15, 16, IEEE PGnet 2011, 2012, IEEE FMEC 2016, WPMC 2017, CSCN 2017 and ANTS 2017 conferences. $\mathrm{He}$ is currently serving as a reviewer for IEEE Communications Letters, IEEE ACCESS, Computers and Electrical Engineering (Elsevier), Journal of Network and Computer Applications (Elsevier), Wireless Networks (Springer) journal, and the Journal of Communications and Networks. . He is also serving as a Guest Editor of Future Generation Computer Systems (Elsevier) and IEEE ACCESS. He is the active member of Pakistan Computer Society and Internet Society Pakistan. 